\title{
Preoperative Weight Loss in the Context of a Comprehensive Lifestyle Intervention
}

\author{
Melissa Kalarchian • Marsha Marcus
}

Received: 31 August 2009/Accepted: 24 September 2009/Published online: 16 October 2009

(C) Springer Science + Business Media, LLC 2009

To the Editor

Drs. Wiezer, Jansen, and Thorell note that 6 months seem to be an unnecessarily long period of intervention in our study on preparing patients for weight-loss surgery (R01DK077102; PREP Study, ClinicalTrials.gov NCT00623792). In particular, they question whether this duration is necessary if the goal is to reduce surgical complications. This is an excellent question, which we would like to address.

Although it is almost certainly true that significant weight loss could occur more quickly with aggressive intervention such as a very low energy diet (VLED), the PREP study is not restricted to the question of whether preoperative weight loss reduces perioperative complications. Rather, we are addressing a broader set of aims including whether a comprehensive lifestyle intervention emphasizing behavioral self-management will confer benefits through 2 years after operation. We hypothesize that patients who participate in the intervention will exhibit better postoperative compliance (e.g., adherence to dietary and exercise guidelines), fewer behavior-related complications (e.g., severe and persistent vomiting, dumping syndrome), and lower healthcare utilization (e.g., surgeryrelated outpatient and emergency room visits), relative to those receiving usual preoperative care.

Our approach is adapted from evidence-based lifestyle interventions that have been associated with modest weight losses (5-10\% of initial body weight) and concomitant improvements in health among individuals at high risk (e.g., $[1,2])$. The 6-month duration of the PREP intervention provides for sufficient time to monitor and address eating behaviors such as overconsumption of high-calorie liquids,

M. Kalarchian $(\bowtie) \cdot$ M. Marcus

WPIC,

Pittsburgh, PA, USA

e-mail: kalarchianma@upmc.edu frequent snacking, and recurrent loss of control over eating, which have been associated with complications and poor weight control after bariatric surgery. Furthermore, this duration corresponds minimum duration of physiciansupervised dieting most US insurance companies require prior to approval for surgery. In order to facilitate participation among candidates for weight-loss surgery-many of whom have physical limitations on their mobility or travel a significant distance to seek care - the intervention is delivered through a combination of face-to-face and telephone counseling sessions.

Multidisciplinary work is needed to establish effects of diet, exercise, and behavior change in this patient population as well as to determine the most appropriate timing and duration of preoperative interventions. We were pleased to learn about the SHOPWEL study to evaluate the effect of short-term weight loss 2 to 3 weeks prior to surgery using VLED. We agree that randomized controlled trials currently underway will yield important information for optimizing surgical care.

Melissa A. Kalarchian, Ph.D.

Associate Professor of Psychiatry and Psychology

Marsha D. Marcus, Ph.D.

Professor of Psychiatry and Psychology

Western Psychiatric Institute and Clinic

University of Pittsburgh Medical Center

\section{References}

1. Knowler WC, Barrett-Connor E, Fowler SE, et al. Reduction in the incidence of type 2 diabetes with lifestyle intervention or metformin. N Engl J Med. 2002;346(6):393-403.

2. Look AHEAD Research Group, Pi-Sunyer X, Blackburn G, et al. Reduction in weight and cardiovascular disease risk factors in individuals with type 2 diabetes: one-year results of the look AHEAD trial. Diabetes Care. 2007;30(6):1374-83. Epub 2007 Mar 15. 